\title{
Association between sleep and awake bruxism in patients with migraine
}

\author{
Keryn Sporh Godk ${ }^{1}$ (D) Maria Luiza dos Santos ${ }^{1}$ (D) Marco Antonio Takashi Utiumi ${ }^{2,3,4}$ (D) João Guilherme \\ Bochnia Küster $^{1}$ (D) Luiz Carlos Canalli Filho' ${ }^{1}$ (D) Nikolai José Eustátios Kotsifas ${ }^{1}$ (D) Bin Cheng Tan $^{1}$ \\ Eldislei Mioto $^{1}$ (D) Gabriel Eduardo Faria Colombani' ${ }^{1}$ (D) Elcio Juliato Piovesan ${ }^{2,3}$
}

${ }^{1}$ Federal University of Paraná, Health Sciences Sector, Curitiba PR, Brazil.

${ }^{2}$ Federal University of Paraná General Hospital, Department of Internal Medicine, Curitiba PR, Brazil.

${ }^{3}$ São José Neurology Clinic, São José dos Pinhais PR, Brazil.

${ }^{4}$ Marcelino Champagnat Hospital, Neurology Service, Curitiba PR, Brazil.

\section{$\varnothing$}

Elcio Juliato Piovesan

Rua General Carneiro, 180,

Alto da Glória, Curitiba, Paraná, Brazil

Federal University of Paraná

General Hospital

Zip-Code: 80060-900

Phone number: +5541

33601866

piovesan1@hotmail.com

Edited by:

Marcelo Moraes Valença

Keywords:

Sleep bruxism

Awake bruxism

Migraine Disorders

Impact profile of the disease

Depression

Comorbidity

\begin{abstract}
Introduction

When migraine undergoes transformation from episodic to chronic form it becomes more disabling due to the refractoriness in treatment and the emergence of comorbidities, with the establishment of a bidirectional relationship between sleep bruxism and chronic migraine. This study aimed to assess whether sleep and awake bruxism are more prevalent in chronic migraine when compared to episodic migraine and also to establish possible clinical correlations with the process of chronification.

Methods

210 patients were allocated to the study, 97 with episodic migraine and 113 with chronic migraine, who underwent face-to-face interviews with the completion of the scales: specific questionnaire for the diagnosis of sleep and awake bruxism, PHQ-9 (depression), GAD-7 (anxiety), Epworth Scale (daytime sleepiness), MIDAS (migraine incapacity) and HIT-6 (impact of headache).

Results

The prevalence of sleep and awake bruxism was similar in patients with episodic versus chronic migraine $(p=0.300$ and $p=0.238$ ). The correlation of patients with concomitant awake and sleep bruxism and with high scores on the migraine incapacity (MIDAS) and headache impact (HIT-6) scales was higher among patients with chronic migraine than in patients with episodic migraine. $(p<0.001$ and $p<0.001)$.

Conclusion

Sleep and awake bruxism alone are not more prevalent in chronic migraine when compared to episodic migraine, although bruxism causes greater impact and disability on individuals with chronic migraine.
\end{abstract}




\section{Introduction}

M igraine is considered one of the most debilitating pathologies with roughly half of the patients losing functional capacity during migraine attacks in addition to being associated with a wide spectrum of comorbidities. ${ }^{1}$ In Brazil, migraine has a prevalence of $15.2 \%$ reaching its peak in the third decade of life and in the female sex (27.1\%). ${ }^{2}$ According to its frequency, it can be classified as episodic (<15 days a month) or chronic ( $\geq 15$ days a month for at least 3 months a year). ${ }^{3}$ Migraine is clinically characterized by a headache with a duration of 4 to 72 hours if left untreated, with a unilateral, pulsatile pain pattern of moderate to severe intensity, aggravated by routine physical activity and usually associated with nausea, vomiting, photophobia, phonophobia and allodynia. ${ }^{4}$ The prevalence of the chronic form of migraine in Brazil is $5.12 \%$ being characterized by a greater impact on the quality of life, more refractoriness to prophylactic treatments, greater predisposition to comorbidities, disability and loss of productivity, and an increased demand for medical services and hospitalizations that consequently generate a high socioeconomic cost. 5,6

Bruxism is a frequent disorder with $85 \%$ to $90 \%$ of the general population reporting at least one episode of grinding or clenching their teeth throughout their lives. ${ }^{7}$ Bruxism is defined as a repetitive activity of the masticatory muscles that is characterized by squeezing or grinding the teeth and/or pushing or holding the jaw, being classified according to its circadian phenotype in sleep bruxism or awake bruxism. ${ }^{8}$ More recently, sleep bruxism was defined as masticatory muscle activity during sleep, formed by a rhythmic phase (Phasic Phase) and a nonrhythmic phase (Tonic Phase). Awake Bruxism is an activity of the masticatory muscles that occurs during wakefulness, being characterized by repetitive and prolonged tooth contact and/or locking or protrusion of the jaw. Both are not considered movement disorders in healthy individuals. ${ }^{9}$ Self-reported sleep bruxism is $13 \%$ in the adult population and for awake bruxism the prevalence is $22 \%$ in adults and $31 \%$ in women and young people. ${ }^{10}$ It is estimated that one in five people in the general population have a clinical overlap between sleep and awake bruxism. ${ }^{11}$ The presence of bruxism can be associated with environmental and genetic factors, stress, anxiety, depression, alterations in the autonomic system, sleep structure, and use of drugs or medications, and those are in line with the factors and comorbidities related to migraine. ${ }^{12,13}$

The association between migraine and bruxism in adults has been documented by several studies, although the causality has not yet been completely elucidated. ${ }^{14} \mathrm{~A}$ study carried out in Brazil in 2013 showed that $74.6 \%$ of participants with chronic migraine also had sleep bruxism. ${ }^{15}$ Canto et al. in 2014 demonstrated that the risk for patients with the chronic form of migraine to develop sleep bruxism is 3.12-3.8 times higher thus demonstrating a bidirectional relationship between those two pathologies. Regarding episodic migraine no statistically significant results were evidenced. ${ }^{16}$

The influence of sleep or awake bruxism in the evolution process of episodic to chronic migraine is not well reported in the literature. This study aimed to assess whether sleep and awake bruxism are more prevalent in chronic migraine when compared to episodic migraine and also to establish possible clinical correlations with chronification.

\section{Methods}

\section{Study design}

A comparative cross-sectional observational study between episodic migraine (EM) and chronic migraine (CM) was carried out, and individuals of both sexes aged between 18 and 64 years participated. Diagnosis of EM and CM was defined according to the criteria of the International Classification of Headache Disorders 3rd edition (ICHD-3). ${ }^{3}$ Patients were allocated into groups based on consultations carried out between 2018 and 2020 and three Brazilian healthcare centers participated in this research: a tertiarylevel healthcare center that exclusively serves the public health system (Federal University of Paraná General Hospital) and two headache outpatient clinics (São José Neurology Clinic and Marcelino Champagnat Hospital).

\section{Ethical aspects}

This study was approved by the Ethics Committee of the Federal University of Paraná General Hospital (registration 2.732.610, CAAE number: 87998518.8.0000.0096) and was registered in the Brazilian Registry of Clinical Trials (RBR-9wgwni). Written Informed Consent was obtained from all the patients prior to data collection.

\section{Study design and population characteristics}

Subjects allocated to study participation should meet the following criteria: (1) Present a definitive diagnosis of EM or CM (with or without analgesic abuse) in accordance with the ICHD-3 criteria; (2) Had migraine attacks within a minimum period of six months at the beginning of the 
study; (3) Had no limitations in information retrieval (e.g., severe aphasia, severe hearing loss, or other situations that could limit the understanding of the questionnaire applied); (4) Had no associated conditions that could promote diagnostic confusion (e.g., HIV infections, active cancer, use of immunosuppressive drugs); (5) Completed all medical questionnaires; and (6) Agreed to participate in the study by signing the Written Informed Consent form. All subjects included in the study received a clinical diagnosis of EM or CM after a face-to-face medical consultation with a neurologist with experience in the area of headache (authors MATU and EJP). The exclusion criteria were: (1) Withdrawal by the participant of the consent to participate in the study; and (2) The development of any other type of headache during the research interval.

Weekly alcohol consumption was classified as: (1) Present; or (2) Absent. The aerobic physical activity classification was in accordance with the World Health Organization recommendations: (1) $\geq 150$ minutes of moderate intensity or (2) $\geq 75$ minutes of vigorous intensity per week were considerated as adequate physical activity and the rest was considered as (3) sedentary. ${ }^{17}$ Monthly income was calculated to assess the socioeconomic influence on the results. The monthly earnings of all family members were added and divided by the number of individuals residing in the family group. For years with migraine disease, the period between the first migraine attack and the time of study evaluation was considered.

\section{Data collection, instruments and methods used}

To assess depression, anxiety and daytime sleepiness, the following scales were respectively used: PHQ-9 (Patient Health Questionnaire-9)18; GAD-7 (Generalized Anxiety Disorder-7) ${ }^{19}$ and Epworth scale. ${ }^{20}$ MIDAS (Migraine Disability Assessment Test) ${ }^{21}$ and HIT-6 (Headache Impact Test) ${ }^{22}$ scales were also included to assess, respectively, migraine disability and headache impact in study subjects.

\section{Identification of awake and sleep bruxism}

To establish the diagnosis of sleep bruxism, a questionnaire consisting of seven questions prepared by the American Academy for Sleep Disorders was used ${ }^{23}$ : (1) "Do you grind or clench your teeth during sleep?"; (2) "When you wake up, do you feel pain or fatigue in the muscles of the face?"; (3) "When you wake up and move your mouth, do you notice stiffness or blockage in your joint?"; (4) "You feel discomfort in your teeth when you wake up?"; (5) "Do you feel a headache in your temples when you wake up?"; (6) "Has a relative or roommate ever reported that you make creaking noises while sleeping?"; (7) "In the past three months, have you had fractured teeth or fillings, except for cavities or leaks?". Using those questions patients were evaluated in the last 30 days prior to treatment using a Likert scale consisting of five possible answers: never; less than once a month; between one and three days a month; between one and three days a week; more than four days a week. Frequent bruxism was considered when the individual reported a frequency equal to or greater than once a week for questions 1 or 6 . When the frequency was less than once a week, but occurring at least once a month, it was considered as eventual bruxism.

To characterize awake bruxism four questions were applied following the Oral Behavior Checklist"24: (1) "Do you grind or clench your teeth when you are awake?"; (2) "Do you press, touch or hold your teeth when not chewing?"; (3) "Do you hold or tense the muscles without chewing?"; (4) "Do you press, touch or hold your teeth when you are not chewing?". The Likert scale was also applied to quantify awake bruxism. Awake bruxism was considered as present when the patient answered questions 1, 2 and/or 3 with the statement "sometime".

\section{Directed acyclic graphs}

Prior to data analysis, directed acyclic graphs were used to demonstrate each of our assumptions and for statistical adjustment (Figures 1A, 1B and 1C). The structured model, based on information from the literature regarding possible relationships between bruxism and migraine, included variables considered relevant for predicting sleep bruxism and $\mathrm{CM}^{25,26}$ (Figure 1A). The second model considered anxiety and depression symptoms as part of a confounding pathway between sleep bruxism and migraine chronicity (Figure 1B). The third model shows our assumptions for the effect of wakefulness bruxism as a factor in the chronicity of migraine (Figure 1C). The directed acyclic graphs were developed in DAGitty software. 

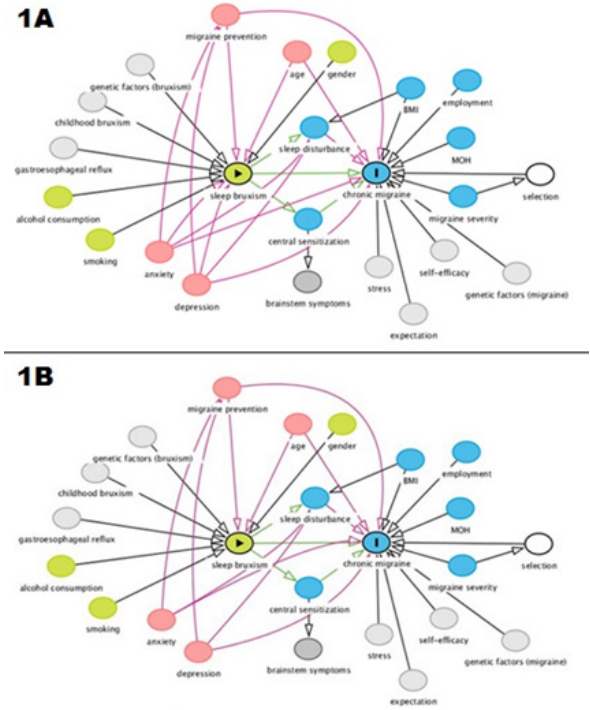

$1 \mathrm{C}$

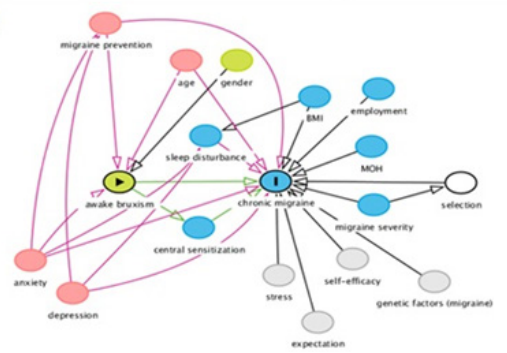

Figure 1. Figures 1A and 1B: Model 1 and 2 (adjusted) for sleep bruxism. Figure 1C: Model 3 for awake bruxism. Circles: green - antecedent factors of bruxism; blue - antecedents of migraine chronicity; red - factors needing adjustment; light gray: factors not measured in this study; green with triangle - bruxism; blue marked with I - chronic migraine; white - selection bias. Arrows: black - causal relationship; green - effects of bruxism on migraine chronification; red - confounding pathway that needs adjustment.

\section{Statistical analysis}

All statistical analyzes were conducted using $R$ version 4.0.2.16. Shapiro-Wilk test and quantile-quantile graphs were used to verify normality. Thus, sample data were summarized as mean \pm standard deviation, median (interquartile range) and count (percentage ratio). A multivariate logistic regression model was fitted according to each model assumption with the presence of $C M$ as the dependent variable to calculate the profile's odds ratio (OR) and likelihood ratio ranges. To assess the model's fit, residual analysis, the ratio between residual deviation and residual degrees of freedom, the Hosmer and Lemeshow test, the Osius-Rojek test, the Stukel test and the influence analysis were used. Tests were performed with a significance level of 0.05 and the listwise exclusion method was used to deal with missing data.

\section{Results}

254 individuals were invited to participate in the study, of which 212 (83\%) agreed. After the interview, two patients were excluded after presenting a recent headache pattern different from migraine. Thus, 97 patients (46\%) with a diagnosis of EM and 113 patients (54\%) with CM were included. Of the group of patients with EM, 76 did not have aura (78\%) and 21 had aura (22\%). In the CM group 78 patients (69\%) had analgesic abuse.

The EM and $C M$ groups did not differ with regards to age $(p=0.187)$, sex $(p=0.746)$, marital status $(p=0.451)$, race $(p=0.167)$, routine physical activity $(p=0.480)$, body mass index $(p=0.446)$, family income $(p=0.131)$ and smoking $(p=0.191)$. Patients with EM had higher alcohol consumption than patients with $C M(p=0.020)$. Patients with $C M$ had a slightly longer duration of disease, but not significant ( $p=0.093$ ). Analgesic abuse was higher in the CM group $(p<0.001)$. The degree of disability was also higher in the CM group (MIDAS score) $(p<0.001)$ (Table 1).

Table 1. Descriptive and comparative analysis of general clinical aspects in the episodic and chronic migraine groups.

\begin{tabular}{|c|c|c|c|}
\hline Variable & Episodic migraine ( $n=97$ ) & Chronic migraine $(n=113)$ & $p$-value \\
\hline Age (years) & $38.21 \pm 12.54$ & $40.54 \pm 12.67$ & 0.187 \\
\hline Gender: female & $88(91 \%)$ & $101(89 \%)$ & 0.746 \\
\hline Marital status: married & $56(58 \%)$ & $71(63 \%)$ & 0.451 \\
\hline Skin color: white & $79(81 \%)$ & $83(73 \%)$ & 0.167 \\
\hline MIDAS score & $20(39)$ & $57(73)$ & $<0.001^{* * *}$ \\
\hline Migraine duration (years) & $10(14.75)$ & $13(18)$ & 0.093 \\
\hline Analgesic abuse & $24(25 \%)$ & 78 (69\%) & $<0.001^{* * *}$ \\
\hline $\begin{array}{c}\text { Adequate physical } \\
\text { activity } \dagger\end{array}$ & $20(21 \%)$ & $19(17 \%)$ & 0.480 \\
\hline BMI $\left(\mathrm{kg} / \mathrm{m}^{2}\right)$ & $25.32(6.73)$ & $25.49(7.43)$ & 0.446 \\
\hline $\begin{array}{c}\text { Monthly household income } \\
\text { per resident } \\
\text { (Brazilian real) }\end{array}$ & $2500(2500)$ & $1500(1500)$ & 0.131 \\
\hline Current or former smoker & $3(3 \%)$ & $9(8 \%)$ & 0.191 \\
\hline $\begin{array}{l}\text { Weekly alcohol } \\
\text { consumption }\end{array}$ & $24(25 \%)$ & $14(12 \%)$ & $0.02^{*}$ \\
\hline
\end{tabular}

All data are summarized as mean \pm standard deviation, count (frequency, $\%)$ or median (interquartile ratio) according to the variable type and distribution. * $p<0.050$; ${ }^{* *} p<0.010$; ${ }^{* * *} p<0.001$. † At least 150 minutes of moderate-intensity or 75 minutes of vigorous-intensity aerobic physical activity. MIDAS: Migraine Disability Assessment.

The frequency of sleep and awake bruxism was similar in the EM and CM groups ( $p=0.300$ and $p=0.238$ ). Anxiety and depression scores were higher in the $C M$ group ( $p=0.012$ and $p=0.003$, respectively). The daytime sleepiness score was similar in the EM and CM groups 
(Table 2). There was no significant difference when comparing the effect of the presence of isolated sleep or awake bruxism with the absence of bruxism in patients with CM on the MIDAS and HIT-6 scores (Table 3).

Table 2. Descriptive and comparative analysis of sleep and awake bruxism in the episodic and chronic migraine groups.

\begin{tabular}{l|c|c|c}
\multicolumn{1}{c|}{ Variable } & Episodic migraine (n=97) & Chronic migraine (n=113) & -value \\
\hline Sleep bruxism: absent & $30(31 \%)$ & $29(26 \%)$ & 0.300 \\
Sleep bruxism: eventual & $25(26 \%)$ & $23(20 \%)$ & - \\
Sleep bruxism: frequent & $42(43 \%)$ & $61(54 \%)$ & - \\
Awake bruxism: absent & $33(34 \%)$ & $39(35 \%)$ & 0.238 \\
Awake bruxism: eventual & $46(47 \%)$ & $43(38 \%)$ & - \\
Awake bruxism: frequent & $18(19 \%)$ & $31(27 \%)$ & - \\
GAD-7 (anxiety) & $8(8)$ & $11(9)$ & $0.012^{*}$ \\
PHQ-9 (depression) & $7(7)$ & $10(8)$ & $0.003^{* *}$ \\
Epworth Scale (daytime & $6(7)$ & $6(8)$ & 0.807 \\
sleepiness)
\end{tabular}

All data are summarized as count (frequency, \%) or median (interquartile ratio) according to the variable type and distribution. ${ }^{*} \mathrm{p}<0.050$; ** $\mathrm{p}<0.010 ;{ }^{* * *} \mathrm{p}<0.001$. GAD-7: Generalized Anxiety Disorder-7. PHQ-9: Patient Health Questionnaire-9.

When compared with patients with CM without bruxism, those with both sleep and awake bruxism had a worse disability measured by MIDAS ( $p=0.003$ ), whereas the impact of headache by the HIT-6 scale was similar with or without bruxism ( $p=0.210)$. However, when the HIT-6 scale was measured at different degrees of impact, a statistically significant difference was found ( $p=0.007$ ).

Among patients with both types of bruxism, $92.4 \%$ had a severe headache impact score, while patients without bruxism had a percentage of $72.7 \%$ (Table 3). For EM the difference between the MIDAS and HIT-6 scores regarding the absence and presence of the two types of bruxism alone or together was not significant.

When comparing the $E M$ versus $C M$ groups, regarding the effect of sleep bruxism on MIDAS and HIT-6, no statistical differences were found $(p=0.126$ and $p=0.310$, respectively). Regarding the effect of awake bruxism on MIDAS and HIT-6 the results obtained were also not statistically different ( $p=0.930$ and $p=0.220$, respectively). In the association of both bruxisms, it was observed that the difference was significant regarding the effect of bruxism on the MIDAS and HIT-6 scores being more pronounced in the CM group ( $p<0.001$ and $p<0.001$ ) (Table 4).

Table 3. Relationship between MIDAS and HIT-6 scores and sleep bruxism, awake bruxism and both in the chronic migraine group.

\begin{tabular}{|c|c|c|c|c|}
\hline & & \multicolumn{3}{|l|}{ Chronic migraine } \\
\hline & & With sleep bruxism & $\begin{array}{l}\text { Without sleep } \\
\text { bruxism }\end{array}$ & p-value \\
\hline \multirow[t]{4}{*}{ MIDAS } & Grade 1 & $4(25 \%)$ & $5(22.7 \%)$ & \multirow{4}{*}{0.800} \\
\hline & Grade 2 & 0 & $2(9.1 \%)$ & \\
\hline & Grade 3 & $2(12.5 \%)$ & $2(9.1 \%)$ & \\
\hline & Grade 4 & 10 (62.5\%) & 13 (59.1\%) & \\
\hline Midas score & & $32,5(8.75 ; 122.5)$ & $30(6.750 ; 66.5)$ & 0.600 \\
\hline \multirow[t]{4}{*}{ HIT-6 } & Little or no impact & 0 & $1(4.5 \%)$ & \multirow{4}{*}{0.740} \\
\hline & Moderate impact & $1(6.3 \%)$ & $4(18.2 \%)$ & \\
\hline & Substantial impact & $1(6.3 \%)$ & $1(4.5 \%)$ & \\
\hline & Severe impact & $14(87.5 \%)$ & $16(72.7 \%)$ & \\
\hline \multirow[t]{2}{*}{ HIT-6 score } & & $66,5(60.75 ; 72)$ & $65(57.25 ; 68.75)$ & 0.520 \\
\hline & & With awake bruxism & $\begin{array}{c}\text { Without awake } \\
\text { bruxism }\end{array}$ & \\
\hline \multirow[t]{4}{*}{ MIDAS } & Grade 1 & $1(20 \%)$ & $5(22.7 \%)$ & \multirow{4}{*}{0.400} \\
\hline & Grade 2 & 0 & $2(9.1 \%)$ & \\
\hline & Grade 3 & $2(40 \%)$ & $2(9.1 \%)$ & \\
\hline & Grade 4 & $2(40 \%)$ & $13(59.1 \%)$ & \\
\hline Midas score & & $15(11 ; 76)$ & $30(6.75 ; 66.5)$ & 0.970 \\
\hline \multirow[t]{4}{*}{ HIT-6 } & Little or no impact & 0 & $1(4.5 \%)$ & \multirow{4}{*}{1} \\
\hline & Moderate impact & $1(20 \%)$ & $4(18.2 \%)$ & \\
\hline & Substantial impact & 0 & $1(4.5 \%)$ & \\
\hline & Severe impact & $4(80 \%)$ & $16(72.7 \%)$ & \\
\hline \multirow[t]{2}{*}{ HIT-6 score } & & $66(66 ; 66)$ & $65(57.25 ; 68.75)$ & 1 \\
\hline & & $\begin{array}{c}\text { Both sleep and awake } \\
\text { bruxism }\end{array}$ & Without bruxism & \\
\hline \multirow[t]{4}{*}{ MIDAS } & Grade 1 & $5(7.6 \%)$ & $5(22.7 \%)$ & \multirow{4}{*}{0.060} \\
\hline & Grade 2 & $2(3 \%)$ & $2(9.1 \%)$ & \\
\hline & Grade 3 & $4(6.1 \%)$ & $2(9.1 \%)$ & \\
\hline & Grade 4 & 55 (83.3\%) & 13 (59.1\%) & \\
\hline Midas score & & $69(35 ; 101)$ & $30(6.75 ; 66.5)$ & $0.003^{* *}$ \\
\hline \multirow[t]{4}{*}{ HIT-6 } & Little or no impact & 0 & $1(4.5 \%)$ & \multirow{4}{*}{$0.007 * *$} \\
\hline & Moderate impact & $1(1.5 \%)$ & $4(18.2 \%)$ & \\
\hline & Substantial impact & $4(6.1 \%)$ & $1(4.5 \%)$ & \\
\hline & Severe impact & $61(92,4 \%)$ & $16(72.7 \%)$ & \\
\hline HIT-6 score & & $66(64 ; 70)$ & $65(57.25 ; 68.75)$ & 0.210 \\
\hline
\end{tabular}

All data are summarized as count (frequency, \%) or median (interquartile ratio) according to the variable type and distribution. ${ }^{*} \mathrm{p}<0.050$; ** $\mathrm{p}<0.010$; *** $\mathrm{p}<0.001$. MIDAS: Migraine Disability Assessment. HIT-6: Headache Impact Test. 
Table 4. Relationship between MIDAS and HIT-6 scores and sleep bruxism, awake bruxism and both in episodic migraine versus chronic migraine.

\begin{tabular}{|c|c|c|c|c|}
\hline & Episodic migraine & Chronic migraine & \multirow{2}{*}{ p-value } \\
\hline & & With sleep bruxism & With sleep bruxism & \\
\hline \multirow{4}{*}{ MIDAS } & Grade 1 & $3(33.3 \%)$ & $4(25 \%)$ & \multirow{4}{*}{$0.048^{*}$} \\
\hline & Grade 2 & $3(33.3 \%)$ & 0 & \\
\hline & Grade 3 & $1(11.1 \%)$ & $2(12.5 \%)$ & \\
\hline & Grade 4 & $2(22.2 \%)$ & $10(62.5 \%)$ & \\
\hline \multirow[t]{2}{*}{ Midas score } & & $7(5 ; 17)$ & $32,5(8.75 ; 122.5)$ & \multirow[t]{2}{*}{0.126} \\
\hline & Little or no impact & 0 & 0 & \\
\hline \multirow{3}{*}{ HIT-6 } & Moderate impact & $2(22.2 \%)$ & $1(6.3 \%)$ & \multirow{2}{*}{0.460} \\
\hline & Substantial impact & $1(11.1 \%)$ & $1(6.3 \%)$ & \\
\hline & Severe impact & $6(66.7 \%)$ & $14(87.5 \%)$ & \multirow{3}{*}{0.310} \\
\hline \multirow[t]{2}{*}{ HIT-6 score } & & $66(59 ; 67)$ & $66,5(60.75 ; 72)$ & \\
\hline & & With awake bruxism & $\begin{array}{l}\text { With awake } \\
\text { bruxism }\end{array}$ & \\
\hline \multirow{4}{*}{ MIDAS } & Grade 1 & $2(28.6 \%)$ & $1(20 \%)$ & \multirow{4}{*}{1} \\
\hline & Grade 2 & $1(14.3 \%)$ & 0 & \\
\hline & Grade 3 & $1(14.3 \%)$ & $2(40 \%)$ & \\
\hline & Grade 4 & $3(42.9 \%)$ & $2(40 \%)$ & \\
\hline \multirow[t]{2}{*}{ Midas score } & & $15(6 ; 37)$ & $15(11 ; 76)$ & \multirow[t]{2}{*}{0.930} \\
\hline & Little or no impact & 0 & 0 & \\
\hline \multirow{3}{*}{ HIT-6 } & Moderate impact & $2(28.6 \%)$ & $1(20 \%)$ & \multirow{2}{*}{2} \\
\hline & Substantial impact & $1(14.3 \%)$ & 0 & \\
\hline & Severe impact & $4(57.1 \%)$ & $4(80 \%)$ & \multirow{3}{*}{0.220} \\
\hline \multirow[t]{2}{*}{ HIT-6 score } & & $62(55 ; 64)$ & $66(66 ; 66)$ & \\
\hline & & $\begin{array}{c}\text { Both sleep and awake } \\
\text { bruxism }\end{array}$ & $\begin{array}{l}\text { Both sleep and } \\
\text { awake bruxism }\end{array}$ & \\
\hline \multirow{4}{*}{ MIDAS } & Grade 1 & $9(16.7 \%)$ & $5(7.6 \%)$ & \multirow{4}{*}{$0.002 * *$} \\
\hline & Grade 2 & $7(13 \%)$ & $2(3 \%)$ & \\
\hline & Grade 3 & $10(18.5 \%)$ & $4(6.1 \%)$ & \\
\hline & Grade 4 & $28(51.9 \%)$ & $55(83.3 \%)$ & \\
\hline \multirow[t]{2}{*}{ Midas score } & & $22,5(10 ; 58)$ & $69(35 ; 101)$ & \multirow[t]{2}{*}{$<0.001^{* * *}$} \\
\hline & Little or no impact & $3(5.6 \%)$ & 0 & \\
\hline \multirow{3}{*}{ HIT-6 } & Moderate impact & $5(9.3 \%)$ & $1(1.5 \%)$ & \multirow{3}{*}{$0.020^{*}$} \\
\hline & Substantial impact & $6(11.1 \%)$ & $4(6.1 \%)$ & \\
\hline & Severe impact & $40(74.1 \%)$ & 61 (92.4\%) & \\
\hline HIT-6 score & & $63(59.25 ; 66.75)$ & $66(64 ; 70)$ & $<0.001^{* * *}$ \\
\hline
\end{tabular}

All data are summarized as count (frequency, \%) or median (interquartile ratio) according to the variable type and distribution. * $\mathrm{p}<0.050$; ${ }^{* *}$ $p<0.010 ;{ }^{* * *} p<0.001$. MIDAS: Migraine Disability Assessment. HIT-6: Headache Impact Test.

For patients with both types of bruxism, a difference was found in the MIDAS classifications between patients in the two groups (EM and $C M$ ), and for patients with $C M$, $83.3 \%$ were classified as 4 , while for EM this proportion was of $51.9 \%(p=0.002)$. A statistical difference was also found between the categories of the HIT-6 questionnaire, with the CM group having $92.4 \%$ of patients classified as severe impact, while for patients with EM this proportion was $74.1 \%(p=0.020)$ (Table 4$)$.

Using multivariate logistic regression to estimate the effect of bruxism on migraine, four scenarios were separated, according to the adjustment of the variables, but there was no difference in the prevalence of bruxism between the EM and CM groups (Table 5).

Table 5. Multivariate logistic regression models for estimating the effect of bruxism on migraine.

$\begin{array}{ccccc}\text { Model } & \text { Comparison } & \text { OR } & 95 \% \mathrm{Cl} \\ \text { Not adjusted } & \text { Sleep bruxism: absent } & \text { Ref. } & - \\ & \text { Sleep bruxism: eventual } & 0.95 & 0.440-2.040 \\ & \text { Sleep bruxism: frequent } & 1.50 & 0.790-2.870 \\ \text { Model 1 } & \text { Sleep bruxism: absent } & \text { Ref. } & - \\ & \text { Sleep bruxism: eventual } & 1.17 & 0.520-2.680 \\ & \text { Sleep bruxism: frequent } & 1.50 & 0.750-3.000 \\ \text { Model 2 } & \text { Sleep bruxism: absent } & \text { Ref. } & - \\ & \text { Sleep bruxism: eventual } & 1.15 & 0.500-2.650 \\ & \text { Sleep bruxism: frequent } & 1.35 & 0.660-2.750 \\ \text { Model 3 } & \text { Awake bruxism: absent } & \text { Ref. } & - \\ & \text { Awake bruxism: eventual } & 0.77 & 0.390-1.520 \\ & \text { Awake bruxism: frequent } & 1.33 & 0.580-3.060\end{array}$

Odds ratio for chronic migraine to cause more bruxism in different models for those with sleep bruxism (models 1 and 2) and awake bruxism (model 3). Model 1 was adjusted for age, use of preventive medication for migraine and selection method. Model 2 added adjustments for anxiety and depression symptoms. Model 3 was adjusted for age, anxiety symptoms, use of preventive medication for migraine and selection method. OR: Odds ratio. $\mathrm{Cl}$ : Confidence interval. Ref.: reference level.

\section{Discussion}

We have observed in the literature that patients with EM and $C M$ have an increased prevalence of sleep bruxism and awake bruxism. ${ }^{14,16}$ No previous study aimed to assess the risk that sleep and/or awake bruxism could cause in the process of chronification of migraine. However, a study carried out in Brazil showed that the association of sleep bruxism with migraine was significant although only in the chronic form, with no significant association in the episodic form of the disease..$^{15}$ However, data from our study did not support sleep or awake bruxism as being more prevalent in CM when compared to EM, which could indicate that there is no relationship between bruxism and migraine chronicity. However when sleep and awake bruxism presented concomitally we observed greater impact and disability on individuals with $C M$.

As demonstrated by the results of this study, CM is a disease with greater debilitating power than the episodic form. This disease brings together patients from different groups of individuals, as it has in common a headache pattern typical of migraine, but at the same time they have 
symptoms that are not related to headache and that can identified as comorbidities. The CaMEO study was perhaps one of the most important efforts to identify and classify patients with $\mathrm{CM}$ into different groups based on their other associated clinical characteristics (comorbidities). ${ }^{27}$ In this study, according to the group of symptoms (comorbidities), patients were subdivided into eight classes: Class 1 Multimorbidities (several associated comorbidities); Class 2 - Respiratory and Psychiatric Comorbidities; Class 3 Respiratory and other pains (eg fibromyalgia); Class 4 Respiratory; Class 5 - Psychiatric; Class 6 - Cardiovascular; Class 7 - Pain; Class 8 - Few Comorbidities.

This classification allowed a risk stratification for the evolution of EM to CM. Thus, patients with Class 1 have an annual risk of 5.34 times higher to undergo migraine chronification. On the other hand, patients with only one comorbidity have a 1.53 times greater risk of suffering this transformation than migraine individuals without comorbidities. ${ }^{28}$ In these studies, awake and/or sleep bruxism were not evaluated as a factor in the evolution of EM to CM.

Another fact from these studies was that the assessment of the impact of migraine using the MIDAS scales proved to be effective as a prognostic factor of chronification, which when high influences any of the reported classes of comorbidities..$^{27,28}$ In the present study, it was observed that individuals with sleep and awake bruxism at the same time have a higher degree of migraine disability. Given these data, we can assume that bruxism could collaborate with the degree of disability in CM.

The disability caused by migraine does not fully explain the association between the comorbidity classes and the risk of progression to the chronic form, demonstrating that multiple comorbidities may play a role in this transformation process but only a higher degree of disability alone would not justify the transformation from EM to $C M .{ }^{28}$ There is, therefore, the need for an aggregating factor of transformation, as may have been the case with the presence of both types of bruxism. A 10-point increase in disability (MIDAS) is believed to have the power to transform EM in CM by 1.11 times, 20 points 1.22 times, and 40 points 1.49 times. ${ }^{28}$

An European study evaluated the correlation of the impact of headache (HIT-6) with sleep bruxism diagnosed by polysomnography. It has been shown that the relationship between sleep bruxism and the impact of headache on the patient's life is only modest, being altered only in patients with phasic bruxism and is associated with the moment of awakening. ${ }^{29}$ In our study, however, we did not find significant relevance of the effect of isolated sleep bruxism on the degree of impact of headache in patients with EM and CM. However, there was a positive association of a higher headache impact score in patients with $C M$ who had both sleep and awake bruxism. In the literature, there are no reports of similar studies that trace the relationship of the impact of headache (HIT-6) with the chronicity of migraine, as well as whether bruxism would have any influence on it. In other words, analyzing these data together, it is possible to show that patients affected by both forms of bruxism could have repercussions on the severity of CM when assessed by the degree of impact of the headache.

A limitation found in our study was that the diagnosis of bruxism is only classified as possible, as it was based on self-report, without clinical evaluation and complementary tests which are necessary for a probable and definitive diagnosis. ${ }^{8}$ A healthy control group was also not formed to be used in the comparisons. Future studies using longitudinal and controlled methodology would be useful to elucidate the influences of sleep and awake bruxism on both forms of migraine.

\section{Conclusion}

In conclusion, sleep or awake bruxism alone are not more prevalent in CM when compared to EM. We observed, however, that bruxism causes greater impact and disability on individuals with CM and thus could participate as a cofactor in the process of migraine chronification.

Conflict of Interest: There is no conflict of interest to declare.

Funding: This study was financed in part by the Coordenação de Aperfeiçoamento de Pessoal de Nível Superior - Brazil (CAPES) - Finance Code 001 (grant number 88887.465414/2019-00). This work was sponsored by Allergan (grant number PG-2020-10985). The funders had no role in study design, data collection and analysis, decision to publish, or preparation of the manuscript.

Authors' contributions: All authors contributed equally to this work.

Keryn Sporh Godk

https://orcid.org/0000-0003-3231-6061

Maria Luiza dos Santos

https://orcid.org/0000-0001-7745-8739

Marco Antonio Takashi Utiumi

https://orcid.org/0000-0001-5273-6798

João Guilherme Bochnia Küster

https://orcid.org/0000-0002-1828-2726

Luiz Carlos Canalli Filho

https://orcid.org/0000-0001-5438-2823 
Nikolai José Eustátios Kotsifas

https://orcid.org/0000-0003-4735-1812

https://orcid.org/0000-0003-0812-7906

Eldislei Mioto

https://orcid.org/0000-0001-5376-9292

Gabriel Eduardo Faria Colombani

https://orcid.org/0000-0003-2774-5152

Elcio Juliato Piovesan

https://orcid.org/0000-0002-0915-0430
Bin Cheng Tan

an international consensus. J Oral Rehabil 2013;40(1):24 Doi: 10.1111 /joor. 12011

10. Ella B, Ghorayeb I, Burbaud P and Guehl D. Bruxism in Movement Disorders: A Comprehensive Review. J Prosthodont 2017;26(7):599-605 Doi: 10.1111/ jopr. 12479

11. Lavigne GJ, Khoury S, Abe S, Yamaguchi T and Raphael $\mathrm{K}$. Bruxism physiology and pathology: an overview for clinicians. J Oral Rehabil 2008;35(7):476-494 Doi: 10.1111/j.1365-2842.2008.01881.x

12. Wieckiewicz M, Paradowska-Stolarz A and Wieckiewicz W. Psychosocial aspects of bruxism: the most paramount factor influencing teeth grinding. Biomed Res Int 2014;469187 Doi: 10.1155/2014/469187

1. Brandes JL. Migraine and functional impairment. CNS Drugs 2009;23(12):1039-1045 Doi: 10.2165/11530030-000000000-00000

2. Queiroz LP, Peres MF, Piovesan EJ, Kowacs F, Ciciarelli MC, Souza JA and Zukerman E. A nationwide populationbased study of migraine in Brazil. Cephalalgia 2009;29(6):642-649 Doi: 10.1111/j.14682982.2008.01782.x

3. Headache Classification Committee of the International Headache Society (IHS) The International Classification of Headache Disorders, 3rd edition. Cephalalgia 2018;38(1):1-211 Doi: 10.1177/0333102417738202

4. Ribeiro FAM, Anderle F, Grassi V, Barea LM, Stelzer FG and Reppold CRJRBdNeP. Avaliação Neuropsicológica em Pacientes com Enxaqueca Episódica e Enxaqueca Crônica/Cefaleia Associada ao uso Excessivo de Analgésicos. 2017;21(1):17-32

5. Giacomozzi AR, Vindas AP, Silva AA, Jr., Bordini CA, Buonanotte CF, Roesler CA, . . . Filho PF. Latin American consensus on guidelines for chronic migraine treatment. Arq Neuropsiquiatr 2013;71(7):478-486 Doi: 10.1590/0004-282x20130066

6. Lantéri-Minet $M$, Duru $G$, Mudge $M$ and Cottrell $S$. Quality of life impairment, disability and economic burden associated with chronic daily headache, focusing on chronic migraine with or without medication overuse: a systematic review. Cephalalgia 201 1;31(7):837-850 Doi: 10.1177/0333102411398400

7. Sateia MJ. International Classification of Sleep DisordersThird Edition. Chest 2014;146(5):1387-1394 Doi: 10.1378/chest. 14-0970

8. Lobbezoo F, Ahlberg J, Raphael KG, Wetselaar P, Glaros $A G$, Kato $T, \ldots$ Manfredini $D$. International consensus on the assessment of bruxism: Report of a work in progress. J Oral Rehabil 2018;45(11):837-844 Doi: 10.1111/ joor. 12663

9. Lobbezoo F, Ahlberg J, Glaros AG, Kato T, Koyano K, Lavigne $G$ J, . . Winocur E. Bruxism defined and graded:
13. Manfredini D. The Triangle Bruxism, Pain, and Psychosocial Factors. Academic Centre for Dentistry Amsterdam (ACTA), Amsterdam, The Netherlands; 2021

14. Costa AL, D'Abreu A and Cendes F. Temporomandibular joint internal derangement: association with headache, joint effusion, bruxism, and joint pain. J Contemp Dent Pract 2008;9(6):9-16

15. Fernandes G, Franco AL, Gonçalves DA, Speciali JG, Bigal ME and Camparis CM. Temporomandibular disorders, sleep bruxism, and primary headaches are mutually associated. J Orofac Pain 2013;27(1):14-20 Doi: 10.11607/jop.921

16. De Luca Canto G, Singh V, Bigal ME, Major PW and Flores-Mir C. Association between tension-type headache and migraine with sleep bruxism: a systematic review. Headache 2014;54(9):1460-1469 Doi: 10.1111/ head. 12446

17. Organization WH. Global Recommendations on Physical Activity for Health. 2010;60p

18. Kroenke K, Spitzer RL and Williams JB. The PHQ-9: validity of a brief depression severity measure. J Gen Intern Med 2001; 16(9):606-613 Doi: 10.1046/i. 15251497.2001.016009606.x

19. Spitzer RL, Kroenke K, Williams JB and Löwe B. A brief measure for assessing generalized anxiety disorder: the GAD-7. Arch Intern Med 2006; 166(10): 1092-1097 Doi: https://www.doi.org/10.1001/archinte.166.10.1092

20. Bertolazi AN, Fagondes SC, Hoff LS, Pedro VD, Menna Barreto SS and Johns MW. Portuguese-language version of the Epworth sleepiness scale: validation for use in Brazil. J Bras Pneumol 2009;35(9):877-883 Doi: 10.1590/s 1806-37132009000900009

21. Fragoso YD. MIDAS (Migraine Disability Assessment): a valuable tool for work-site identification of migraine in workers in Brazil. Sao Paulo Med J 2002; 120(4): 118 121 Doi: 10.1590/s1516-31802002000400006

22. Martin M, Blaisdell B, Kwong JW and Bjorner JB. The Short-Form Headache Impact Test (HIT-6) was 
psychometrically equivalent in nine languages. J Clin Epidemiol 2004;57(12):1271-1278 Doi: 10.1016/i. jclinepi.2004.05.004

23. Sateia MJ. International classification of sleep disordersthird edition: highlights and modifications. Chest 2014; 146(5):1387-1394 Doi: 10.1378/chest.14-0970

24. Markiewicz MR, Ohrbach R and McCall WD, Jr. Oral behaviors checklist: reliability of performance in targeted waking-state behaviors. J Orofac Pain 2006;20(4):306316

25. Castroflorio T, Bargellini A, Rossini G, Cugliari G and Deregibus A. Sleep bruxism and related risk factors in adults: A systematic literature review. Arch Oral Biol 2017;83(1)25-32 Doi: 10.1016/i. archoralbio.2017.07.002

26. Probyn K, Bowers H, Caldwell F, Mistry D, Underwood $M$, Matharu $M$ and Pincus T. Prognostic factors for chronic headache:Asystematic review. Neurology 2017;89(3):291301 Doi: 10.1212/wnl.0000000000004112
27. Lipton RB, Fanning KM, Buse DC, Martin VT, Reed ML, Manack Adams A and Goadsby PJ. Identifying Natural Subgroups of Migraine Based on Comorbidity and Concomitant Condition Profiles: Results of the Chronic Migraine Epidemiology and Outcomes (CaMEO) Study. Headache 2018;58(7):933-947 Doi: 10.1111/ head. 13342

28. Lipton RB, Fanning KM, Buse DC, Martin VT, Hohaia LB, Adams AM, . . . Goadsby PJ. Migraine progression in subgroups of migraine based on comorbidities: Results of the CaMEO Study. Neurology 2019;93(24):e2224-e2236 Doi: 10.1212/ wnl.0000000000008589

29. Martynowicz H, Smardz J, Michalek-Zrabkowska M, Gac P, Poreba R, Wojakowska A, . . . Wieckiewicz M. Evaluation of Relationship Between Sleep Bruxism and Headache Impact Test-6 (HIT-6) Scores: A Polysomnographic Study. Front Neurol 2019;10(487 Doi: 10.3389/fneur.2019.00487 